\title{
Schwarzschild Black Hole in Noncommutative Spaces
}

\author{
Forough Nasseri \\ Department of Physics, Sabzevar University of Tarbiat Moallem, P.O. Box 397, Sabzevar, Iran \\ Khayyam Planetarium, P.O. Box 769, Neishabour, Iran
}

(November 23, 2018)

\begin{abstract}
We study the effects of noncommutative spaces on the horizon, the area spectrum and Hawking temperature of a Schwarzschild black hole. The results show deviations from the usual horizon, area spectrum and the Hawking temperature. The deviations depend on the parameter of space/space noncommutativity.
\end{abstract}

\section{Introduction}

Recently, remotivated by string theory arguments, noncommutative spaces (Moyal plane) have been studied extensively. The noncommutative space can be realized by the coordinate operators satisfying

$$
\left[\hat{x}_{\mu}, \hat{x}_{\nu}\right]=i \theta_{\mu \nu}
$$

where $\hat{x}$ are the coordinate operators and $\theta_{\mu \nu}$ is the noncommutativity parameter and is of dimension (length) ${ }^{2}$; for a review on the string theory side, see [1]. In noncommutative spaces, the usual product of fields should be replaced by the star-product:

$$
(f \star g)=\left.\exp \left(\frac{i}{2} \theta_{\mu \nu} \frac{\partial}{\partial x^{\mu}} \frac{\partial}{\partial y^{\nu}}\right) f(x) g(y)\right|_{x=y},
$$

where $f$ and $g$ are two arbitrary infinitely differentiable functions on $R^{3+1}$. For noncommutative spacetime $\left(\theta_{0 i} \neq 0\right)$ it has been shown that the theory is not unitary and hence, as a field theory, it is not appealing $[2,3]$. In noncommutative spaces we have

$$
\left[\hat{x}_{i}, \hat{x}_{j}\right]=i \theta_{i j}, \quad\left[\hat{x}_{i}, \hat{p}_{j}\right]=i \delta_{i j}, \quad\left[\hat{p}_{i}, \hat{p}_{j}\right]=0 .
$$

In this letter we focus on the metric of a Schwarzschild black hole in a noncommutative space. Given the metric and assuming that the noncommutative parameter $\left(\theta_{i j}\right)$ is small, we study the horizon, 
the area spectrum and the Hawking temperature of the Schwarzschild black hole. Since the noncommutativity in space violates rotational symmetry, our horizon corrections have a preferred direction. We will use a natural unit system that sets $k_{B}, c$, and $\hbar$ all equal to 1 , so that $\ell_{P}=M_{P}^{-1}=\sqrt{G}$.

The plan of this letter is as follows. In section 2, we present the horizon, area spectrum and Hawking temperature of the Schwarzschild black hole in noncommutative spaces. We discuss our results and conclude in Sec. 3.

\section{Noncommutativity and Schwarzschild black hole}

The metric of the Schwarzschild black hole is given by

$$
d s^{2}=\left(1-\frac{2 G M}{r}\right) d t^{2}-\frac{d r^{2}}{\left(1-\frac{2 G M}{r}\right)}-r^{2}\left(d \theta^{2}+\sin ^{2} \theta d \phi^{2}\right) .
$$

There is a horizon at $r_{\mathrm{h}}=2 G M$. The horizon area $A$ of the Schwarzschild black hole is given by

$$
A=r_{\mathrm{h}}^{2} \int_{0}^{2 \pi} d \phi \int_{0}^{\pi} \sin \theta d \theta=4 \pi r_{\mathrm{h}}^{2}=16 \pi G^{2} M^{2} .
$$

The Hawking temperature of the Schwarzschild black hole is given by

$T_{\mathrm{H}}=\kappa /(2 \pi)$ where $\kappa$ is the surface gravity of the black hole [4]. For Schwarzschild black hole $\kappa=G M / r_{\mathrm{h}}^{2}$ and therefore

$$
T_{\mathrm{H}}=\frac{G M}{2 \pi r_{\mathrm{h}}^{2}}=\frac{1}{8 \pi G M} \text {. }
$$

We propose the following metric for the Schwarzschild black hole in the noncommutative spaces:

$$
d s^{2}=\left(1-\frac{2 G M}{\sqrt{\hat{r} \hat{r}}}\right) d t^{2}-\frac{d \hat{r} d \hat{r}}{\left(1-\frac{2 G M}{\sqrt{\hat{r} \hat{r}}}\right)}-\hat{r} \hat{r}\left(d \theta^{2}+\sin ^{2} \theta d \phi^{2}\right),
$$

where $\hat{r}$ satisfying (3). The horizon of the noncommutative metric (7) satisfies the following condition

$$
1-\frac{2 G M}{\sqrt{\hat{r} \hat{r}}}=0
$$


Now, we note that there is a new coordinate system,

$$
x_{i}=\hat{x}_{i}+\frac{1}{2} \theta_{i j} \hat{p}_{j}, \quad p_{i}=\hat{p}_{i},
$$

where the new variables satisfy the usual canonical commutation relations:

$$
\left[x_{i}, x_{j}\right]=0, \quad\left[x_{i}, p_{j}\right]=i \delta_{i j}, \quad\left[p_{i}, p_{j}\right]=0 .
$$

So, if in the horizon condition (8) we change the variables $\hat{x}_{i}$ to $x_{i}$, the horizon of the noncommutative metric (7) satisfies the following condition

$$
1-\frac{2 G M}{\sqrt{\left(x_{i}-\theta_{i j} p_{j} / 2 \hbar\right)\left(x_{i}-\theta_{i k} p_{k} / 2 \hbar\right)}}=0 .
$$

This leads us to

$$
1-\frac{2 G M}{r}-G M \frac{x_{i} \theta_{i j} p_{j}}{r^{3}}+\mathcal{O}\left(\theta^{2}\right)=0,
$$

or

$$
1-\frac{2 G M}{r}-G M \frac{L \cdot \theta}{2 r^{3}}+\mathcal{O}\left(\theta^{2}\right)=0,
$$

where $\theta_{i j}=\frac{1}{2} \epsilon_{i j k} \theta_{k}, L=r \times p$. If we put $\theta_{3}=\theta$ and the rest of the $\theta$ components to zero (which can be done by a rotation or a redefinition of coordinates), then $L . \theta=L_{z} \theta$. So, we can rewrite Eq. (13) as

$$
r^{3}-2 G M r^{2}-\frac{G M L_{z} \theta}{2}=0 .
$$

By the following definitions

$$
a \equiv-2 G M, \quad c \equiv-\frac{G M L_{z} \theta}{2},
$$

the real root of cubic formula (14) is the horizon of the Schwarzschild black hole in noncommutative spaces

$$
\begin{aligned}
\hat{r}_{h} & \equiv-\frac{a}{3}+\left(\frac{-2 a^{3}-27 c+\sqrt{108 a^{3} c+729 c^{2}}}{54}\right)^{1 / 3} \\
& +\left(\frac{-2 a^{3}-27 c-\sqrt{108 a^{3} c+729 c^{2}}}{54}\right)^{1 / 3} .
\end{aligned}
$$


Two other roots of cubic formula (14) are not real [5]. In the case of commutative spaces $c=0$, Eq. (16) yields $r_{h}=2 G M$. The Hawking temperature and the horizon area of Schwarzschild black hole in noncommutative spaces are respectively

$$
\begin{aligned}
T_{H} & =\frac{G M}{2 \pi \hat{r}_{h} \hat{r}_{h}}, \\
A & =4 \pi \hat{r}_{h} \hat{r}_{h} .
\end{aligned}
$$

Substituting $\hat{r}_{h}$ from (16) into (17) and (18) give us the Hawking temperature and the horizon area of the Schwarzschild black hole in noncommutative spaces.

\section{Conclusion}

In this letter, we investigate the effects of noncommutative spaces on the Schwarzschild black hole. We calculate the horizon, the area spectrum and the Hawking temperature in the noncommutative spaces. Our results show deviations from the usual formulae. The deviations depend on the parameter of space/space noncommutativity.

\section{References}

[1] N. Seiberg and E. Witten, JHEP 09 (1999) 032.

[2] J. Gomis and T. Mehen, Nucl. Phys. B 591 (2000) 265.

[3] M. Chaichian, et. al., Eur. Phys. J. C 20 (2001) 767.

[4] N.D. Birrell and P.C.W. Davies, "Quantum Fields in Curved Space", Cambridge University Press (1982).

[5] http://planetmath.org/encyclopedia/CubicFormula.html. 